\title{
The triglyceride glucose index (TyG) and CDKAL1 gene rs10946398 SNP are associated with NAFLD in Chinese adults
}

Jun Zhu

xuzhou central hospital

Dujuan Xu

Xuzhou central hospital

Ruihua Yang

xuzhou central hospital

Min Liu

xuzhou central hospital

Ying Liu ( $\nabla$ ziqiangbuxi314@126.com )

xuzhou central hospital

\section{Research}

Keywords: Triglyceride glucose index, Non-alcoholic fatty liver disease, CDKAL1 gene

Posted Date: April 2nd, 2020

DOl: https://doi.org/10.21203/rs.3.rs-20248/v1

License: (9) This work is licensed under a Creative Commons Attribution 4.0 International License. Read Full License

Version of Record: A version of this preprint was published at Minerva Endocrinology on December 1st, 2020. See the published version at https://doi.org/10.23736/S0391-1977.20.03273-3. 


\section{Abstract}

Background: Non-alcoholic fatty liver disease (NAFLD) has a higher prevalence worldwide, and its pathogenesis is not clear. Genetic factors, dyslipidaemia and dysglycaemia have been proven to be associated with NAFLD. It has not been reported whether the triglyceride glucose index (TyG), which is estimated by triglyceride and fasting glucose, has a relationship with NAFLD in people from North China. Whether the CDKAL1 gene rs10946398 SNP, which has been found to be associated with BMI, has a relationship with TyG and NAFLD is not clear.

Methods: We recruited a total of 1,760 subjects in this study, and we measured the clinical characteristics, abdominal ultrasound, and genotype of those participants. Results: The results showed that $527(29.9 \%)$ subjects suffered from NAFLD, the TyG index was associated with NAFLD (OR=5.456, 95\% Cl (3.526 8.442)), and the CDKAL1 gene rs10946398 SNP has a relationship with NAFLD (OR=1.509, 95\% $\mathrm{Cl}(1.046 \sim 2.178)$ ). The distribution of the $\mathrm{C}$ allele of $\mathrm{rs} 10946398$ was statistically significant at different levels of the TyG index.

Conclusion: We identified an association between the rs10946398 genotypes of CDKAL1 and NAFLD and the TyG index, and the TyG index was related to the risk of NAFLD.

\section{Background}

Non-alcoholic fatty liver disease (NAFLD) has become a public health issue worldwide. In Western countries, approximately $30 \% \sim 40 \%$ of men and $15 \%$ 20\% of women suffer from NAFLD[1]. Jian GF et al. [2] reported that the prevalence of NAFLD in Asia is approximately $25 \%$, similar to that in Europe. In China, there may be some regional differences in the prevalence of NAFLD. The prevalence is only $14.7 \%$ in northwest China, but in economically developed areas, such as Shenzhen city, the prevalence of NAFLD is $30.75 \%[3]$. The increasing morbidity of NAFLD aggravates the burden of people, and the pathological mechanisms of NAFLD are not clear. Some papers have reported that insulin resistance is closely related to NAFLD[4-5]. Dyslipidaemia and genetic factors were proven to be associated with NAFLD[6-7].

The cyclin-dependent kinase 5 (CDK5) regulatory subunit-associated protein 1-like 1 (CDKAL1) gene is located on chromosome 6p22.3 and encodes a 65-kD protein. Several studies identified that the CDKAL1 gene was associated with type 2 diabetes[8], and Liang et al.[9] found that this relationship existed between the CDKAL1 gene rs10946398 single nucleotide polymorphism (SNP) and insulin resistance. In addition, Wen et al.[10] proved that the rs10946398 SNP was associated with BMI. BMI and insulin resistance are risk factors for NAFLD. Therefore, we hypothesized that there is a relationship between the CDKAL1 rs10946398 SNP and NAFLD.

Currently, the triglyceride glucose index (TyG), which is estimated by triglyceride and fasting glucose, has been proven to be related to metabolic syndrome and cardiovascular disease[11-12]. Several studies have considered that the TyG index is a surrogate marker of insulin resistance. Lee SB et al.[13] found that the TyG index is superior to the homeostasis model assessment of insulin resistance (HOMA-IR) for 
predicting NAFLD in Korea. However, few studies have expounded the association between the TyG index and NAFLD in Chinese adults. Therefore, in the present study, we sought to explore the relationship between NAFLD and the TyG index and the association between the CKDAL1 rs10946398 SNP and NAFLD in Chinese adults.

\section{Methods}

Study population

Initially, a total of 2,044 subjects who underwent physical examination at Xuzhou Central Hospital from Jan. 2015 to Oct. 2016 were selected as participants. Each participant, who were all from Xuzhou city, North China, had a complete medical examination, blood laboratory tests, ultrasonographic reports and oral glucose tolerance tests (OGTT). The medical history, alcohol consumption, and drug intake details of the participants were recorded. Participants with alcohol intake greater than $20 \mathrm{~g} / \mathrm{d}$ were defined as having a "habit of drinking alcohol", and those participants were excluded. Patients with diabetes, hypertriglyceridaemia, and subjects taking special drugs, such as anti-diabetic and lipid-lowering drugs, were excluded from this study. Individuals with acute inflammation, cancer, or liver disease, such as viral hepatitis or liver cirrhosis, were also excluded from the present study. Finally, 1,760 participants (26 87 years old) were included in the analyses. This study was reviewed and approved by the ethics committee of Xuzhou Central Hospital, China, and informed consent was signed by all subjects.

Ultrasonographic analyses

All subjects were checked for abdominal ultrasonography by two doctors who were blind to the participant's clinical information. A diagnosis of fatty liver disease was made on the basis of the findings of an abdominal ultrasonography scan performed using a 3.5-MHz transducer (LOGIQ9E, GE, USA), and two doctors had the same estimate on one individual.

Genotype determination

A QIAamp Blood Mini Kit (Qiagen, Chatsworth, CA, USA) was used to extract genomic DNA from the buffy coat fraction of centrifuged blood. The SNP rs10946398 in the CDKAL1 gene was selected because some literature has reported that this genotype is associated with obesity and T2DM in the East Asian population[. ABI 7900HT (Applied Biosystems, Foster City, CA, USA) was used to determine the rs10946398 genotypes with TaqMan SNP allelic discrimination.

Clinical characteristics of the study participants

Height and weight were measured in all subjects, and body mass index (BMI) was estimated by dividing the weight in kilograms by the height in metres squared. Blood pressure was measured after resting for 10 min. Waist circumference (WC) was measured with the participants standing naturally, feet separated by $25 \mathrm{~cm} \sim 30 \mathrm{~cm}$. The WC is the horizontal circumference through the centre of the umbilicus, measured 
with a soft ruler at the end of expiration and before inhalation. These anthropometric parameters were measured by trained doctors.

Blood samples were collected after fasting for $8 \sim 10 \mathrm{~h}$ from all subjects. The samples were allowed to clot at less than $25^{\circ} \mathrm{C}$ for $1 \sim 3 \mathrm{~h}$ after drawing. Immediately following clotting, the samples were subsequently stored at $-80^{\circ} \mathrm{C}$ until further analysis. Biochemical parameters, including fasting plasma glucose (FPG), glutamine transferase (GGT), total cholesterol (TC), high-density lipoprotein cholesterol (HDL-C), low-density lipoprotein cholesterol (LDL-C), serum uric acid (SUA) and fasting insulin, were measured by an autoanalyzer (Type 7600, Hitachi Ltd., Tokyo, Japan). Glycosylated haemoglobin was measured by high-performance liquid chromatography with a standardized method. The serum sample of a standard 75-g OGTT was drawn 120 min after glucose load, but before that, the individual had to fast more than $10 \mathrm{~h}$. Homoeostasis model assessment for insulin resistance (HOMA-IR) was estimated by the formula HOMA-IR $=$ FPG $(\mathrm{mmol} / \mathrm{L}) \star$ fasting insulin $(\mathrm{mU} / \mathrm{mL}) / 22.5$. The TyG index was calculated using the formula TyG $=$ FPG $(\mathrm{mmol} / \mathrm{L}) * T G(\mathrm{mmol} / \mathrm{L}) / 2$.

Statistical analysis

The means \pm standard deviation were used to represent continuous data, and the number (ratio\%) shows the enumeration data. Skewed variables of continuous data such as TyG, GGT and HOMA-IR were Intransformed before analysis. Hardy-Weinberg equilibrium was used to analyse the distribution of the rs10946398 genotypes. Inter-group comparisons of continuous data were performed by ANOVA or $t$ test, and categorical data were compared using the chi-square test. Logistic regression analysis was used to estimate the odds ratio (OR) and associated 95\% confidence interval (Cl) for NAFLD on the basis of the TyG index and rs10946398 genotypes. A dose-response curve was generated using a generalized linear model to show the relationship between the TyG index and the risk of NAFLD. A multiple scatter plot was used to show the distribution of rs10946398 genotypes at different levels of the TyG index. SPSS for Windows version 22.0 (IBM Co., Armonk, NY, USA) and STATA 14.0 (Stata Corp, TX, USA) were used for performing all statistical analyses in the study. Statistical significance was considered for $P$ values less than 0.05 .

\section{Result}

3.1 A total of 1,760 participants were recruited for the present study, including 527 (29.9\%) patients with NAFLD and 1,233 (70.1\%) non-NAFLD persons. The clinical characteristics of all participants are shown in Table 1. The participants were divided into two groups for analysis by two independent sample t-tests. The results show that patients with NAFLD are older and fatter. The SBP, DBP, FPG, 2-h OGTT, HbA1c, TC, TG, LDL, SUA, GGT, TyG, and HOMAIR of the NAFLD group were higher than those of participants with no NAFLD, but HDL was lower. Males are more likely to have NAFLD.

3.2 The results of the Hardy-Weinberg equilibrium test were not statistically significant $\left(X^{2}=0.183\right.$, $P=0.668)$, which means that the genotype distribution was balanced. Sex, WC, BMI, SBP, DBP, TG, HDL, 
LDL, and SUA were not different among the three genotypes of rs10946398. The CC(2) genotype had higher FPG, 2-h OGTT, HbA1c, GGT, TyG, and HOMAIR than the other genotypes (Table 2).

3.3 The subjects were divided into three groups on the basis of their TyG levels (T1: InTyG<0.938; T2: 0.938 1.537; and T3: InTyG 21.537). Table 3 shows that the highest TyG group was older; had a higher WC, BMI, SBP, DBP, FPG, 2-h OGTT, TC, TG, LDL, SUA, GGT and HOMAIR; and had lower HDL levels than the other two groups.

3.4 With increasing TyG levels, the risk of NAFLD is also increased. With adjusting for some variables, such as sex, age, BMI, SBP, DBP, SUA, 2-h OGTT, TC, GGT, and rs10946398 genotypes, the association between TyG and NAFLD was also statistically significant. The risk of NAFLD increasing with TyG levels did not change.

3.5 Figure 1 shows that the risk of NAFLD increases with InTyG levels. This trend remains statistically significant regardless of the change in InTyG levels, and when the TyG levels are higher, the trend is clearer.

3.6 Subjects with the CC allele of rs10946398 are more likely to suffer from NAFLD. Even with adjustment for other factors, this trend remained statistically significant (Table 5, $P=0.022$ ).

3.7 All subjects were divided into three groups by InTyG levels (first group: InTyG <0.938; second group: InTyG:0938 1.537; third group: InTyG $\geq 1.537$ ). We found that the CC genotype of rs10946398 had a higher percentage in the third group (42.4\%), and the AA genotype of rs10946398 had a higher percentage in the first group (36.5\%). Figure 2 shows that the distribution of the $\mathrm{C}$ allele has statistical significance in different groups.

3.8 The association between TyG levels and the risk of NAFLD is statistically significant and increases with the $\mathrm{C}$ allele of rs10946398. After further adjustment for other factors (Model 3), TyG also had a significantly positive association with NAFLD (Table 6).

\section{Discussion}

In the present study, a total of 1,760 Chinese subjects were finally analysed, 527 (29.9\%) of whom were diagnosed with NAFLD by abdominal ultrasonography. Further adjusting for confounding factors, we found that the CDKAL1 rs10946398 SNP was associated with NAFLD, and with an increase in the TyG index, the risk of NAFLD was increased. We also found that rs10946398 genotypes had different distributions among the three levels of the TyG index.

In this study, the results indicated that the $C$ allele of the rs10946398 SNP not only increased the risk of NAFLD but also enhanced the TyG index (Fig. 3).

In addition, it was found that a higher TyG index corresponded to a higher risk of NAFLD in our results. We hypothesized that the association between the rs 10946398 genotypes and NAFLD may be caused by 
the SNP being a BMI-associated gene. Wen et al. reported that rs10946398 was associated with BMI in Asian but not European populations[10]. Most studies have defined a strong correlation between BMI and NAFLD[14-15]. The obesity population has a higher prevalence of NAFLD than the general population, and lean people have the lowest prevalence of NAFLD in Asia[16]. Rs10946398 genotypes of CDKAL1 regulate the CDK5 protein, which is a threonine protein kinase that is expressed in the brain and muscle. CDK5 is active when the A allele of the rs 10946398 genotype of CDKAL1 reduces expression. The activity of CDK5 leads to decreased insulin secretion and increased insulin resistance (IR), which elevates circulating glucose levels. Excess glucose can be converted to fatty acids and finally into fat, and fat accumulates in the liver to generate fatty liver[17-18]. The above may be a reason why the rs 10946398 SNPs of CDKAL1 have a relationship with NAFLD. This speculation was supported by several studies, such as that by Jun Liang et al., who thought that the CDKAL1 gene is associated with metabolic disease by its effect on glucose-related traits[9], Chen et al. confirmed that this gene accounts for the risk of cardiovascular disease[19], and Akiyama M reported that the CDKAL1 gene is associated with obesity[20].

The strong correlation between the TyG index and BMI has been confirmed by previous literature[21-22], but the relationship between BMI-associated genes and the TyG index has not been completely elucidated, especially in Chinese adults. In the present study, we found that there was an independent correlation between the BMI-associated gene CDKAL1 and the TyG index, and the TyG index was calculated by fasting glucose and triglycerides. Table 2 shows that people with the CC genotype of rs10946398 have higher FPG, 2-h OGTT and HbA1c levels than the other two genotypes, indicating that the genotype of rs 10946398 was associated with the TyG index. This relationship is because the $C$ allele of rs10946398 elevates the glucose indexes.

In addition to rs 10946398 related to NAFLD, the TyG index also has an independent association with NAFLD. This result was in agreement with previous studies; for example, Zhang $R$ et al. [23] found that the TyG index could predict the incidence of NAFLD in a prospective cohort study, and some scholars showed that the TyG index was an effective biomarker to identify NAFLD[24]. The change in the TyG index was due to glucose and lipids, and its levels were elevated, indicating the disorder of glucose or lipids. Pathoglycaemia and dislipidaemia are traits of metabolic disease, especially in patients with NAFLD, whose FPG and TG are higher than those of the general population. Our results show that patients with NAFLD have higher TG, LDL-C, FPG and TC levels than non-NAFLD subjects and that there is a dose-response relationship between the TyG index and the risk of NAFLD. The HOMA-IR and TyG index are representative markers of IR. IR, which is the basis of type 2 diabetes, has been shown to have an important pathological relationship with NAFLD[25].

The limitations of this study were as follows. First, the present study is a cross-sectional observational study. We can describe the association between rs 10946398 and the TyG index and NAFLD, but causality cannot be confirmed from these results. Second, the participants of this study were all Chinese Han adults, and most of them were from one city of Xuzhou. Finally, we did not perform histological 
examination of liver biopsy in subjects. Ultrasonography, which is not the gold standard compared with histological examination, is a non-invasive technique with widespread use in clinical practice[26].

\section{Conclusion}

In conclusion, we identified an association between rs10946398 genotypes of CDKAL1 and NAFLD and the TyG index, and the TyG index was related to the risk of NAFLD.

\section{Declarations}

\section{Ethics approval and consent to participate}

This study is reviewed and approved by the ethics committee of Xuzhou central hospital, China, and the informed consent of participant were signed by all subjects. The approved NO. of ethics committee is 2015-02-413.

\section{Consent to publish}

We, all authors, consent to publish this study in Diabetology \&Metabolic syndrome.

\section{Competing interests}

The authors have declared that no competing interests exist.

\section{Availability of data and materials}

All data generated or analyzed during this study are included in this manuscript.

\section{Authors' contributions}

Jun Zhu and Dujuan Xu were the performer of this study and written this manuscript; Ruihua Yang and Min Liu performed the recruitment of patients; Ying Liu and Min Liu were the guarantor of integrity of the entire study, and responsible for the study concepts, study design and approval of the final version of the manuscript; Min Liu was analysis the data onto this study.

\section{Funding}

This work was supported by generous grants from the Natural Science Foundation of Jiangsu Province (BK201711775) and Xuzhou Science and Technology Bureau project (8457) to YL.

\section{Acknowledgements}

We acknowledge and thank all participants for their cooperation and sample contributions. 


\section{References}

1.

Byrne CD, Targher G. NAFLD: a multisystem disease[J]. Journal of hepatology. 2015;62(1):47-64.

2.

Fan JG, Kim SU, Wong, V W S. New trends on obesity and NAFLD in Asia[J]. Journal of hepatology. 2017;67(4):862-73.

3.

Zhu JZ, Zhou QY, Wang YM, et al. Prevalence of fatty liver disease and the economy in China: A systematic review[J]. World journal of gastroenterology: WJG. 2015;21(18):5695.

4.

Martin-Rodriguez JL, Gonzalez-Cantero J, Gonzalez-Cantero A, et al. Insulin resistance and NAFLD: Relationship with intrahepatic iron and serum TNF-a using 1H MR spectroscopy and MRI[J]. Diabetes Metab. 2019;45(5):473-9.

5 .

Fujii H, Imajo K, Yoneda M, et al. HOMA-IR: An independent predictor of advanced liver fibrosis in nondiabetic non-alcoholic fatty liver disease[J]. Journal of gastroenterology hepatology. 2019;34(8):1390-5.

6.

Dongiovanni P, Meroni M, Baselli G, et al. PCSK7 gene variation bridges atherogenic dyslipidemia with hepatic inflammation in NAFLD patients[J]. Journal of lipid research. 2019;60(6):1144-53. 7.

Dowla S, Aslibekyan S, Goss A, et al. Dyslipidemia is associated with pediatric nonalcoholic fatty liver disease[J]. J Clin Lipidol. 2018;12(4):981-7.

8.

Dehwah MA, Wang M, Huang QY. CDKAL1 and type 2 diabetes: a global meta-analysis[J]. Genet Mol Res. 2010;9(2):1109-20.

9.

Liang J, Pei Y, Liu X, et al. The CDKAL 1 gene is associated with impaired insulin secretion and glucoserelated traits: the Cardiometabolic Risk in Chinese (CRC) study[J]. Clin Endocrinol. 2015;83(5):651-5. 10.

Wen W, Cho YS, Zheng W, et al. Meta-analysis identifies common variants associated with body mass index in east Asians[J]. Nat Genet. 2012;44:307-11.

11.

Navarro-González D, Sánchez-Íñigo L, Pastrana-Delgado J, et al. Triglyceride-glucose index (TyG index) in comparison with fasting plasma glucose improved diabetes prediction in patients with normal fasting glucose: the Vascular-Metabolic CUN cohort[J]. Preventive medicine. 2016;86:99-105.

12.

Vasques ACJ, Novaes FS, de Oliveira MS, et al. TyG index performs better than HOMA in a Brazilian population: a hyperglycemic clamp validated study[J]. Diabetes Res Clin Pract. 2011;93(3):98-100. 
13.

Lee SB, Kim MK, Kang S, et al. Triglyceride Glucose Index Is Superior to the Homeostasis Model Assessment of Insulin Resistance for Predicting Nonalcoholic Fatty Liver Disease in Korean Adults[J]. Endocrinology Metabolism. 2019;34(2):179-86.

14.

Liu M, Wang J, Zeng J, et al. Association of NAFLD with diabetes and the impact of BMI changes: a 5year cohort study based on 18,507 elderly[J]. The Journal of Clinical Endocrinology Metabolism. 2017;102(4):1309-16.

15.

Targher G, Byrne CD. Metabolically healthy obesity and NAFLD[J]. Nature Reviews Gastroenterology Hepatology. 2016;13(8):442-4.

16.

Fan JG, Kim SU, Wong, V W S. New trends on obesity and NAFLD in Asia[J]. Journal of hepatology. 2017;67(4):862-73.

17.

Musso G, Gambino R, Cassader M. Recent insights into hepatic lipid metabolism in non-alcoholic fatty liver disease (NAFLD)[J]. Progress in lipid research. 2009;48(1):1-26.

18.

Ipsen DH, Lykkesfeldt J, Tveden-Nyborg P. Molecular mechanisms of hepatic lipid accumulation in nonalcoholic fatty liver disease[J]. Cell Mol Life Sci. 2018;75(18):3313-27.

19.

Chen G, Xu Y, Lin Y, et al. Association study of genetic variants of 17 diabetes-related genes/loci and cardiovascular risk and diabetic nephropathy in the Chinese She population. Journal of Diabetes. 2013;5:136-45.

20.

Akiyama M, Okada Y, Kanai M, et al. Genome-wide association study identifies 112 new loci for body mass index in the Japanese population[J]. Nat Genet. 2017;49(10):1458-64.

21.

Lambrinoudaki I, Kazani MV, Armeni E, et al. The TyG index as a marker of subclinical atherosclerosis and arterial stiffness in lean and overweight postmenopausal women[J]. Heart Lung Circulation. 2018;27(6):716-24.

22.

Khan SH, Sobia F, Niazi NK, et al. Metabolic clustering of risk factors: evaluation of Triglyceride-glucose index (TyG index) for evaluation of insulin resistance[J]. Diabetol Metab Syndr. 2018;10(1):74-7. 23.

Zheng R, Du Z, Wang M, Mao Y, Mao W. A longitudinal epidemiological study on the triglyceride and glucose index and the incident nonalcoholic fatty liver disease. Lipids Health Dis. 2018;17:262-8. 24.

Zhang S, Du T, Zhang J, et al. The triglyceride and glucose index (TyG) is an effective biomarker to identify nonalcoholic fatty liver disease[J]. Lipids Health Dis. 2017;16(15):1-8. 
25.

Hossain IA, Shah MMR, Rahman MK, et al. Gamma glutamyl transferase is an independent determinant for the association of insulin resistance with nonalcoholic fatty liver disease in Bangladeshi adults: association of GGT and HOMA-IR with NAFLD[J]. Diabetes \& Metabolic Syndrome: Clinical Research \& Reviews, 2016, 10(1): S25-9.

26.

Loria P, Adinolfi LE, Bellentani S, et al. Practice guidelines for the diagnosis and management of nonalcoholic fatty liver disease: A decalogue from the Italian Association for the Study of the Liver (AISF) Expert Committee[J]. Digestive Liver Disease. 2010;42(4):272-82.

\section{Tables}

Table 1 The clinical characteristics of all participants according to the status of NAFLD

\begin{tabular}{|c|c|c|c|c|c|}
\hline & All $(n=1760 \square I$ & NAFLD $(n=123$ & NAFLD $(n=527)$ & $\mathrm{t}$ & $\mathrm{P}$ \\
\hline Age (years) & $46.90 \pm 10.09$ & $46.49 \pm 10.07$ & $47.86 \pm 10.09$ & -2.612 & 0.009 \\
\hline Sex (male) & 1340 & 872 & 468 & $66.445^{b}$ & $<0.001$ \\
\hline WC (cm) & $87.24 \pm 9.36$ & $84.43 \pm 8.64$ & $93.82 \pm 7.52$ & -21.399 & $<0.001$ \\
\hline BMI $\left(\mathrm{kg} / \mathrm{m}^{2}\right)$ & $24.65 \pm 3.07$ & $23.73 \pm 2.72$ & $26.82 \pm 2.73$ & -21.842 & $<0.001$ \\
\hline SBP (mmHg) & $124.43 \pm 16.00$ & $121.72 \pm 15.50$ & $130.79 \pm 15.36$ & -11.281 & $<0.001$ \\
\hline DBP (mmHg) & $79.44 \pm 11.58$ & $77.47 \pm 11.25$ & $84.05 \pm 11.03$ & -11.301 & $<0.001$ \\
\hline FPG $(\mathrm{mmol} / \mathrm{L})$ & $5.29 \pm 1.25$ & $5.14 \pm 1.15$ & $5.63 \pm 1.41$ & -7.681 & $<0.001$ \\
\hline 2-h OGTT & $7.58 \pm 3.17$ & $7.17 \pm 2.69$ & $8.63 \pm 3.86$ & -8.533 & $<0.001$ \\
\hline HbA1c (\%) & $5.85 \pm .086$ & $5.74 \pm 0.77$ & $6.11 \pm 0.98$ & -8.582 & $<0.001$ \\
\hline TC & $5.08 \pm 0.91$ & $4.98 \pm 0.87$ & $5.32 \pm 0.96$ & -7.258 & $<0.001$ \\
\hline TG & $1.77 \pm 1.75$ & $1.40 \pm 1.12$ & $2.64 \pm 2.69$ & -14.393 & $<0.001$ \\
\hline HDL & $1.22 \pm 0.29$ & $1.27 \pm 0.30$ & $1.09 \pm 0.24$ & 12.668 & $<0.001$ \\
\hline LDL & $3.01 \pm 0.78$ & $2.97 \pm 0.75$ & $3.11 \pm 0.84$ & -3.411 & 0.001 \\
\hline SUA & $310.98 \pm 78.59$ & $294.28 \pm 71.87$ & $350.05 \pm 79.84$ & -14.414 & $=0.001$ \\
\hline $\mathrm{GGT}^{\mathrm{a}}$ & $3.20 \pm 0.67$ & $3.04 \pm 0.62$ & $3.59 \pm 0.61$ & -17.21 & $<0.001$ \\
\hline $\mathrm{TyG}^{\mathrm{a}}$ & $1.29 \pm 0.70$ & $1.08 \pm 0.61$ & $1.76 \pm 0.65$ & -21.054 & $=0.001$ \\
\hline HOMAIR $^{\mathrm{a}}$ & $0.63 \pm 0.60$ & $0.46 \pm 0.55$ & $1.03 \pm 0.51$ & -20.102 & $<0.001$ \\
\hline
\end{tabular}

Values are expressed as the mean \pm SD or number (\%). TyG, triglyceride glucose; BMI, body mass index; SBP, systolic blood pressure; DBP, diastolic blood pressure; FPG, fasting plasma glucose; TC, total cholesterol; TG, triglyceride; HDL-C, high-density lipoprotein cholesterol; LDL-C, low-density lipoprotein cholesterol; WC, waist circumference; HOMA-IR, homeostasis model assessment of insulin resistance; 2-h OGTT, 2-hour oral glucose tolerance test; HbA1c, glycated haemoglobin; SUA, serum urea; GGT, transglutaminase. 
Table 2 The clinical characteristics of participants according to CDKAL1 rs10946398 genotypes

\begin{tabular}{|c|c|c|c|c|c|}
\hline & \multicolumn{3}{|c|}{ CDKAL1 rs10946398 } & \multirow[b]{2}{*}{$F$} & \multirow[b]{2}{*}{$P$ for trend } \\
\hline & $\mathrm{AA}(0)(\mathrm{n}=576 \square$ & $\mathrm{AC}(1)(\mathrm{n}=854)$ & $\mathrm{CC}(2)(\mathrm{n}=330)$ & & \\
\hline Age & $46.18 \pm 9.54$ & $46.80 \pm 10.01$ & $48.41 \pm 11.09$ & 5.22 & 0.005 \\
\hline Sex (male) & 426 & 652 & 262 & $3.452^{\mathrm{b}}$ & 0.178 \\
\hline WC & $87.24 \pm 9.68$ & $87.21 \pm 9.04$ & $87.30 \pm 9.62$ & 0.01 & 0.92 \\
\hline BMI & $24.88 \pm 3.14$ & $24.55 \pm 2.99$ & $24.53 \pm 3.14$ & 2.678 & 0.102 \\
\hline SBP & $124.42 \pm 16.56$ & $124.64 \pm 15.55$ & $123.92 \pm 16.01$ & 0.207 & 0.649 \\
\hline DBP & $79.33 \pm 12.28$ & $79.54 \pm 11.33$ & $79.38 \pm 10.98$ & 0.003 & 0.954 \\
\hline FPG & $5.16 \pm 1.06$ & $5.31 \pm 1.35$ & $5.44 \pm 1.28$ & 10.588 & 0.001 \\
\hline 2-h OGTT & $7.26 \pm 2.66$ & $7.64 \pm 3.41$ & $8.01 \pm 3.36$ & 9.764 & 0.002 \\
\hline HbA1c (\%) & $5.77 \pm 0.70$ & $5.86 \pm 0.92$ & $5.98 \pm 0.91$ & 11.694 & 0.001 \\
\hline TC & $5.03 \pm 0.86$ & $5.08 \pm 0.95$ & $5.17 \pm 0.88$ & 5.310 & 0.021 \\
\hline TG & $1.75 \pm 1.99$ & $1.71 \pm 1.55$ & $1.94 \pm 1.80$ & 2.304 & 0.129 \\
\hline HDL & $1.22 \pm 0.29$ & $1.22 \pm 0.29$ & $1.20 \pm 0.31$ & 0.470 & 0.493 \\
\hline LDL & $2.97 \pm 0.74$ & $3.02 \pm 0.81$ & $3.07 \pm 0.78$ & 3.505 & 0.061 \\
\hline SUA & $311.49 \pm 80.45$ & $310.39 \pm 78.27$ & $311.64 \pm 76.33$ & 0.001 & 0.978 \\
\hline $\mathrm{GGT}^{\mathrm{a}}$ & $3.16 \pm 0.68$ & $3.18 \pm 0.65$ & $3.34 \pm 0.66$ & 15.5 & $<0.001$ \\
\hline $\mathrm{TyG}^{\mathrm{a}}$ & $1.24 \pm 0.70$ & $1.26 \pm 0.69$ & $1.42 \pm 0.70$ & 13.299 & $<0.001$ \\
\hline HOMAIR $^{\mathrm{a}}$ & $0.61 \pm 0.60$ & $0.62 \pm 0.60$ & $0.71 \pm 0.61$ & 5.258 & 0.022 \\
\hline
\end{tabular}

a: Variable was ln transformed; b: chi-square test

Values are expressed as the mean \pm SD or number (\%). TyG, triglyceride glucose; BMI, body mass index; SBP, systolic blood pressure; DBP, diastolic blood pressure; FPG, fasting plasma glucose; TC, total cholesterol; TG, triglyceride; HDL-C, high-density lipoprotein cholesterol; LDL-C, low-density lipoprotein cholesterol; WC, waist circumference; HOMA-IR, homeostasis model assessment of insulin resistance; 2-h OGTT, 2-hour oral glucose tolerance test; HbA1c, glycated haemoglobin; SUA, serum urea; GGT, transglutaminase.

Table 3 The clinical characteristics of participants according to different TyG levels 


\begin{tabular}{|c|c|c|c|c|c|}
\hline & \multicolumn{3}{|c|}{ TyG $^{\mathrm{a}}$} & \multirow[b]{2}{*}{$F$} & \multirow[b]{2}{*}{$P$ for trend } \\
\hline & T1 $(n=587 \square$ & T2 $(n=588)$ & T3 $(n=585)$ & & \\
\hline Age & $45.60 \pm 9.74$ & $47.61 \pm 10.62$ & $47.49 \pm 9.80$ & 10.237 & 0.001 \\
\hline Sex (male) & 345 & 481 & 514 & 136.48 & $<0.001$ \\
\hline WC & $81.78 \pm 8.79$ & $88.33 \pm 8.77$ & $91.69 \pm 7.38$ & 398.87 & $<0.001$ \\
\hline BMI & $23.05 \pm 2.78$ & $24.88 \pm 2.95$ & $26.03 \pm 2.73$ & 326.44 & $<0.001$ \\
\hline SBP & $118.46 \pm 14.32$ & $125.73 \pm 16.23$ & $129.12 \pm 15.53$ & 140.77 & $<0.001$ \\
\hline DBP & $75.22 \pm 10.54$ & $80.29 \pm 11.60$ & $82.82 \pm 11.27$ & 136.38 & $<0.001$ \\
\hline FPG & $4.84 \pm 0.42$ & $5.18 \pm 0.76$ & $5.85 \pm 1.85$ & 214.67 & $<0.001$ \\
\hline 2-h OGTT & $6.43 \pm 1.67$ & $7.31 \pm 2.42$ & $8.96 \pm 4.24$ & 170.05 & $<0.001$ \\
\hline HbA1c (\%) & $5.57 \pm 0.36$ & $5.79 \pm 0.58$ & $6.20 \pm 1.23$ & 179.55 & $<0.001$ \\
\hline $\mathrm{TC}$ & $4.72 \pm 0.79$ & $5.12 \pm 0.79$ & $5.40 \pm 0.99$ & 186.760 & $<0.001$ \\
\hline TG & $0.76 \pm 0.19$ & $1.36 \pm .027$ & $3.18 \pm 2.43$ & 857.78 & $<0.001$ \\
\hline HDL & $1.37 \pm 0.29$ & $1.23 \pm 0.26$ & $1.04 \pm 0.24$ & 472.760 & $<0.001$ \\
\hline LDL & $2.77 \pm 0.68$ & $3.19 \pm 0.68$ & $3.07 \pm 0.91$ & 46.79 & $<0.001$ \\
\hline SUA & $272.25 \pm 65.75$ & $317.79 \pm 70.48$ & $343.00 \pm 81.79$ & 275.48 & $<0.001$ \\
\hline $\mathrm{GGT}^{\mathrm{a}}$ & $2.82 \pm 0.51$ & $3.20 \pm 0.56$ & $3.60 \pm 0.68$ & 513.58 & $<0.001$ \\
\hline HOMAIR $^{\mathrm{a}}$ & $0.27 \pm 0.50$ & $0.64 \pm 0.52$ & $0.99 \pm 0.55$ & 551.24 & $<0.001$ \\
\hline
\end{tabular}

a: Variable was ln transformed; b: chi-square test

Values are expressed as the mean \pm SD or number (\%). TyG, triglyceride glucose; BMI, body mass index; SBP, systolic blood pressure; DBP, diastolic blood pressure; FPG, fasting plasma glucose; TC, total cholesterol; TG, triglyceride; HDL-C, high-density lipoprotein cholesterol; LDL-C, low-density lipoprotein cholesterol; WC, waist circumference; HOMA-IR, homeostasis model assessment of insulin resistance; 2-h OGTT, 2-hour oral glucose tolerance test; HbA1c, glycated haemoglobin; SUA, serum urea; GGT, transglutaminase.

Table 4 The relationship between TyG levels and the risk of NAFLD

\begin{tabular}{|c|c|c|c|c|}
\hline & \multicolumn{3}{|c|}{$\mathrm{TyG}^{\mathrm{a}}$} & \multirow[b]{2}{*}{ P for trend } \\
\hline & $<0.938$ & $0.938 \sim 1.537$ & $>1.537$ & \\
\hline Model 1 & 1 & $4.978(3.454 \sim 7.176)$ & $16.762(11.731 \sim 23.950)$ & $<0.001$ \\
\hline Model 2 & 1 & $2.932(1.980 \sim 4.342)$ & 8.467 (5.780 12.404) & $<0.001$ \\
\hline Model 3 & 1 & 2.126 (1.377 3.283) & $5.456(3.526 \sim 8.442)$ & $<0.001$ \\
\hline \multicolumn{5}{|c|}{ Model 1: unadjusted } \\
\hline
\end{tabular}


Table 5 The relationship between genotype rs10946398 and the risk of NAFLD

\begin{tabular}{|c|c|c|c|c|}
\hline & \multicolumn{3}{|c|}{ Rs10946398 } & \multirow[b]{2}{*}{$\mathrm{P}$ for trenc } \\
\hline & $\mathrm{AA}(0)$ & $\mathrm{AC}(1)$ & $\mathrm{CC}(2)$ & \\
\hline Model 1 & 1 & $1.111(0.878 \sim 1.405)$ & 1.505 (1.126 2.012) & $<0.001$ \\
\hline Model 2 & 1 & $1.323(1.009 \sim 1.734)$ & 1.874 (1.337 2.626) & $<0.001$ \\
\hline Model 3 & 1 & $1.290(0.963 \sim 1.727)$ & 1.509 (1.046 2.178) & 0.022 \\
\hline
\end{tabular}

Model 1: unadjusted

Model 2: adjusted for sex, age and BMI

Model 3: adjusted for sex, age, BMI, SBP, DBP, SUA, 2-h OGTT, TC, SUA, GGT and TyG

Table 6 The association between TyG and NAFLD under different rs10946398 genotypes

\begin{tabular}{cccccccc}
\hline & & \multicolumn{2}{c}{ Model 1 } & & Model 2 & & Model 3 \\
\cline { 3 - 7 } & & OR & $95 \%$ CI & OR & $95 \%$ CI & OR & 95\% CI \\
\hline rs10946398 & AA(0) & 3.811 & $2.882 \sim 5.039$ & 2.597 & $1.907 \sim 3.535$ & 2.010 & $1.420 \sim 2.845$ \\
& AC(1) & 3.753 & $2.993 \sim 4.706$ & 2.909 & $2.259 \sim 3.746$ & 2.318 & $1.755 \sim 3.063$ \\
\hline CC(2) & 4.250 & $2.925 \sim 6.173$ & 3.432 & $2.249 \sim 5.237$ & 2.599 & $1.523 \sim 4.434$ \\
\hline P for trend & & $<0.001$ & & $<0.001$ & & $<0.001$ \\
\hline
\end{tabular}

Model 1: unadjusted

Model 2: adjusted for sex, age and BMI

Model 3: adjusted for sex, age, BMI, SBP, DBP, SUA, 2-h OGTT, TC, SUA and GGT

\section{Figures}




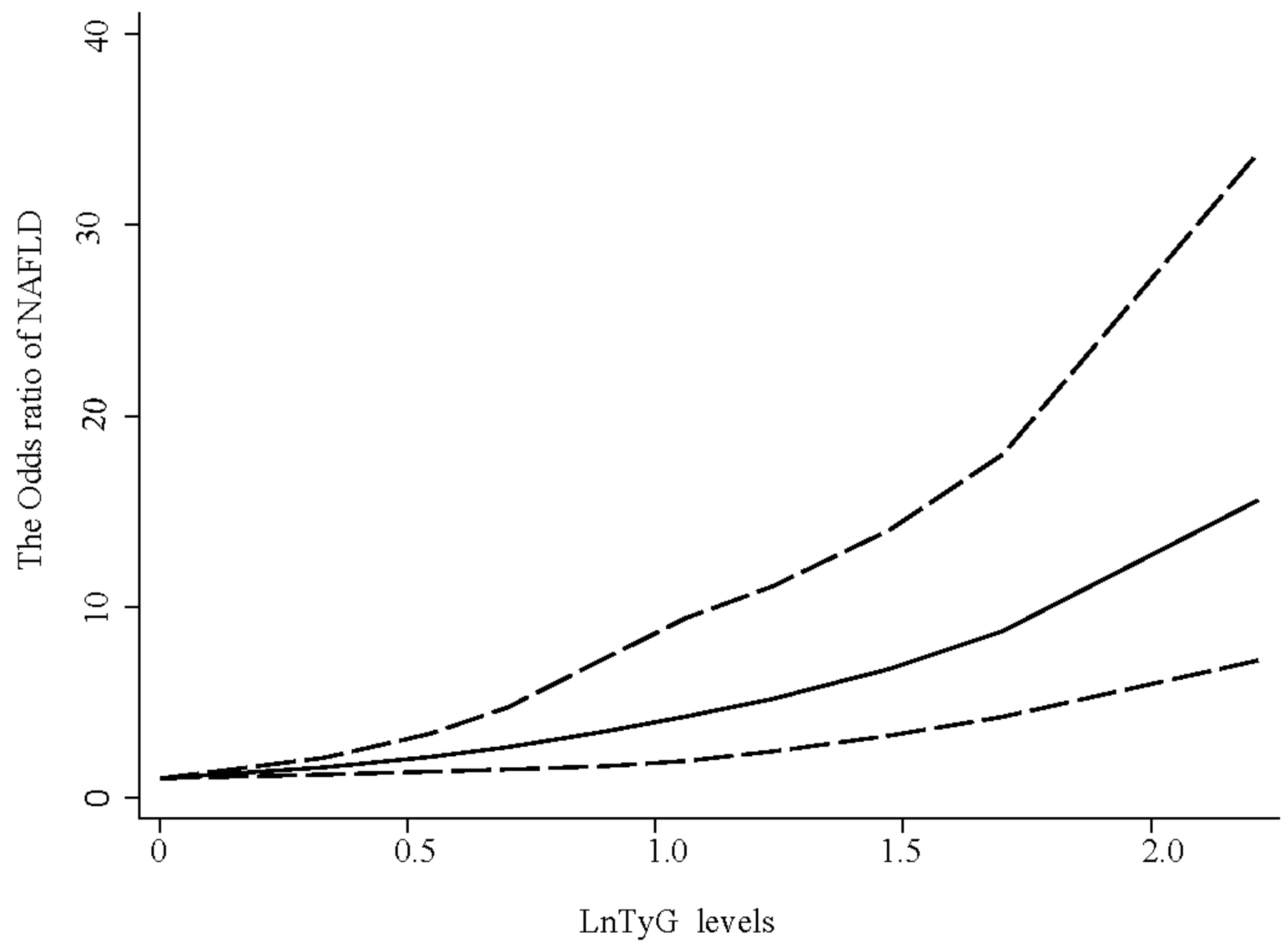

Figure 1

shows that the risk of NAFLD increases with InTyG levels. This trend remains statistically significant regardless of the change in InTyG levels, and when the TyG levels are higher, the trend is clearer. 


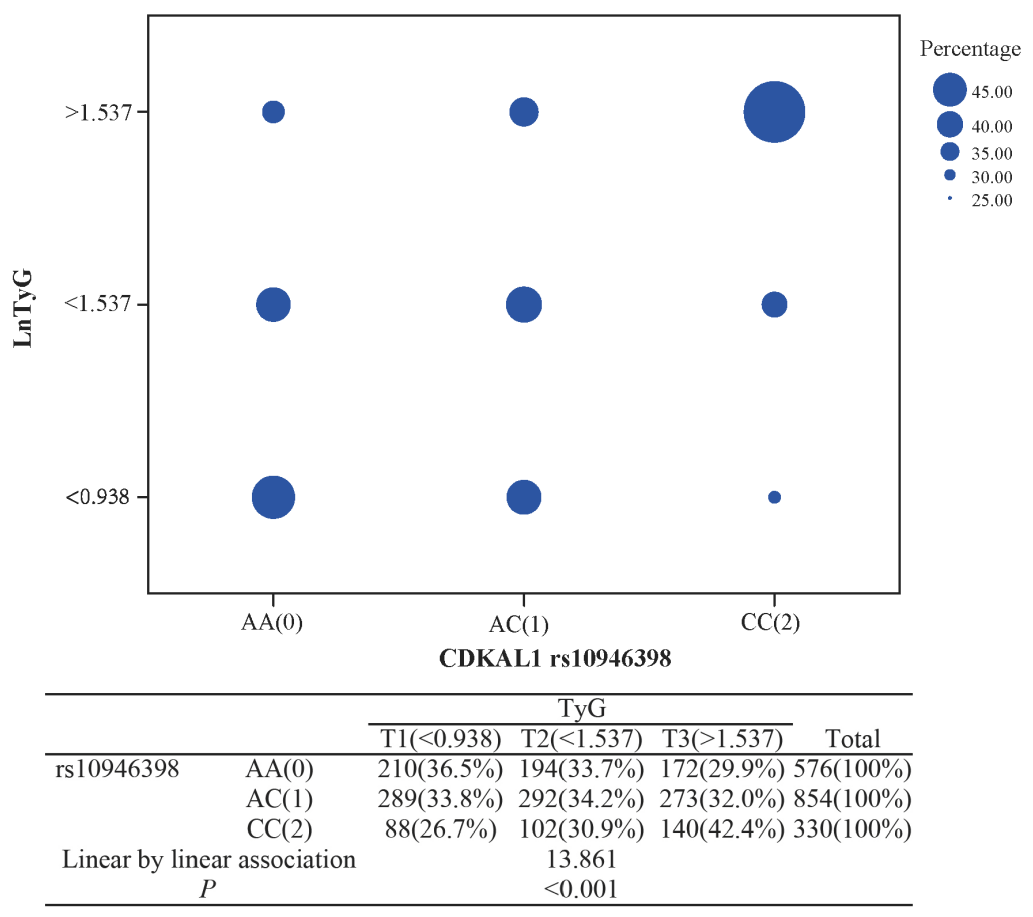

Figure 2

shows that the distribution of the $\mathrm{C}$ allele has statistical significance in different groups. 


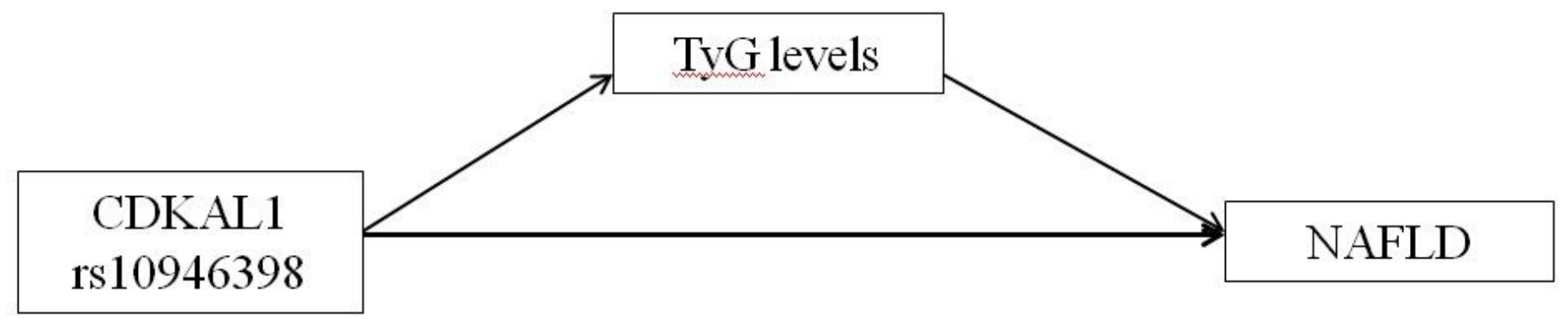

\section{Figure 3}

NAFLD was increased. We also found that rs10946398 genotypes had different distributions among the three levels of the TyG index. In this study, the results indicated that the C allele of the rs10946398 SNP not only increased the risk of NAFLD but also enhanced the TyG index 